\title{
Retroperitoneal teratoma misdiagnosed as a gastric stromal tumor: A case report
}

\author{
LIMING GAN and QIULIN HUANG \\ Department of Gastrointestinal Surgery, The First Affiliated Hospital, University of South China, \\ Hengyang, Hunan 421001, P.R. China
}

Received May 13, 2019; Accepted January 29, 2020

DOI: $10.3892 / \operatorname{mco} .2020 .2019$

\begin{abstract}
The misdiagnosis of retroperitoneal teratoma as a gastric stromal tumor is rarely reported. There are numerous abdominal organs, and retroperitoneal tumors are easily concealed by organs situated more anteriorly on the abdomen; therefore, retroperitoneal tumors are easily misdiagnosed as human digestive system tumors by abdominal surgeons. We herein present the case of a 39-year-old female patient who was diagnosed with a gastric stromal tumor (GST) on preoperative examination, whereas the postoperative diagnosis was retroperitoneal mature cystic teratoma. Such cases are clinically rare. In the present case, the tumor was located in the retroperitoneum, near the lesser gastric curvature, without an obvious gap. The anterior surface of the tumor was concealed by the lesser gastric curvature and did not move significantly with breathing and changes in posture. In such cases, a preoperative misdiagnosis is very likely. The aim of the present study was to improve our understanding of the presentation of retroperitoneal teratomas, thereby gaining more clinical experience and hopefully reducing the rate of misdiagnosis.
\end{abstract}

\section{Introduction}

Teratomas may occur in various parts of the body. They may originate from embryonic and primordial germ cells, embryonic mesenchymal stem cells or embryonic carcinoma cells, and usually contain three embryonic components, which have evolved from the inner, middle and outer primitive embryonic layers (1-3).

Retroperitoneal teratomas are located behind the peritoneum and as such may be obscured by various organs in the abdomen. The majority of patients present with digestive tract

Correspondence to: Professor Qiulin Huang, Department of Gastrointestinal Surgery, The First Affiliated Hospital, University of South China, 69 Chuanshan Road, Hengyang, Hunan 421001, P.R. China

E-mail: 1907722730@qq.com

Key words: retroperitoneal, teratoma, gastric stromal tumor, misdiagnosis symptoms and are easily misdiagnosed. Therefore, retroperitoneal teratoma may be included in the differential diagnosis of gastric stromal tumors and this can be used as a systematic and comprehensive study (4).

The most common method of treatment for retroperitoneal teratomas is surgical. In the present case, surgical resection was performed and the pathological examination revealed a retroperitoneal mature cystic teratoma. Retroperitoneal teratomas are mostly benign, but a few are malignant; therefore, regular re-examination is required in order to optimize postoperative recovery and avoid tumor recurrence (5).

The follow-up results of the present case indicate that the current condition was stable and there was no evidence of recurrence; however, it is necessary to determine whether follow-up should be continued for a long period of time according to the patient's recovery. Therefore, it is crucial for physicians to report and share their experience with the treatment of difficult and/or rare cases $(6,7)$.

\section{Case report}

A 39-year-old female patient was admitted to the Department of Gastrointestinal Surgery of The First Affiliated Hospital of the University of South China with abdominal distension as the main symptom. A laboratory examination revealed mildly elevated levels of $\alpha$-fetoprotein (AFP; $8.230 \mathrm{ng} / \mathrm{ml}$; normal range, $0.0-7.0 \mathrm{ng} / \mathrm{ml}$ ), while other routine examinations did not reveal any significant abnormalities. No abdominal abnormalities were observed on physical examination by the abdominal specialist.

An auxiliary examination raised the suspicion of a gastric stromal tumor (GST) by electronic gastroscopy (Fig. 1) and by dual-phase enhanced computed tomography (CT) of the small intestine (Fig. 2).

According to the patient's medical history and preoperative examination, the proximal tumor and lateral protrusion of the lesser curvature of the stomach were considered to be close to the abdominal aorta; thus, endoscopic submucosal dissection was considered as a high-risk surgical procedure. Following completion of the preoperative preparation, laparoscopy-assisted gastric tumor resection was performed. A $2 \times 2-\mathrm{cm}$ large cystic mass was identified intraoperatively, located close to the abdominal aorta and behind the peritoneum of the lesser curvature of the stomach (Fig. 3). The 
gastric fundus and a bulge of the gastric body were visible during the intraoperative endoscopic examination, and the bulge disappeared when the stomach was lifted; the cystic mass was then completely removed.

Fast frozen biopsy examination revealed that the cyst was $2 \times 1.5 \times 0.3 \mathrm{~cm}$ in size, it was filled with mucus, it had a smooth, pale and soft surface, and the cyst wall had a thickness of $0.1-0.2 \mathrm{~cm}$. Thus, this was suspected to be a mature cystic teratoma. No other mass or protuberance was identified on gastroscopy. The results of the postoperative pathological examination confirmed the diagnosis of retroperitoneal mature cystic teratoma (Fig. 4). Mature cystic teratomas are usually benign and contain several components, including bone, cartilage, teeth, adipose tissue, nerve tissue and other components; in the present case, calcification was also observed (Figs. 2 and 4). The patient was discharged from the hospital without obvious abdominal distension. There was no abdominal distension or other signs or symptoms on the 6-month postoperative follow-up.

\section{Discussion}

A teratoma is a tumor derived from germ cells that may contain multiple types of tissue (1-3). It may develop anywhere in the body, but is most commonly encountered as sacrococcygeal, retroperitoneal, ovarian and testicular teratoma. Teratomas may be classified into three main types, namely immature, mature and monoembryonic. Mature retroperitoneal teratomas are mostly benign and malignant, accounting for $26 \%(8,9)$. The early symptoms of retroperitoneal teratoma may not be readily evident and are non-specific; thus, they are not easily identifiable and the tumor is often misdiagnosed $(10,11)$. Typically, symptoms become more pronounced when the tumor grows to a large size and compresses adjacent tissues or organs $(12,13)$. However, the tumor in the present case was located in the lesser curvature of the stomach and was compressed, resulting in herniation of the lesser curvature of the stomach near the fundus. Preoperative CT examination and ultrasound gastroscopy did not reveal the presence of a tumor, and the abdominal examination revealed no obvious signs. In addition, such cases are rarely reported clinically. For all these reasons, retroperitoneal teratoma may be easily misdiagnosed as a GST (14). In the present case, the abdominal distension had been present for $>10$ years, and there was no recurrence on the 6-month postoperative follow-up. The cause of abdominal distension in this patient was considered to be the compression of the lesser gastric curvature by the tumor.

At its early stages, retroperitoneal teratoma often lacks characteristic clinical manifestations (5). CT and magnetic resonance imaging (MRI) are currently the main imaging examinations for the diagnosis of teratomas. CT scans can determine the size, location, integrity, invasion of adjacent tissues and organs, and the presence of lymph node metastasis. An MRI scan not only clearly indicates the structure of the tumors and surrounding soft tissues and blood vessels, but can also reveal multiple organ structures. MRI is often superior to other examinations for preoperative staging of tumors such as rectal cancer, and the localization and qualitative diagnosis of severe abdominal lesions, as it can provide a favorable preoperative basis for selecting the specific surgical treatment plan for the follow-up (15). Moreover, due to the lack of CT three-dimensional images in the present case, the associations of the tumor with the surrounding organs and structures could not be observed from different angles and levels, and the accurate location of the tumor could not be stereoscopically demonstrated, which may also adversely affect the accuracy of the preoperative diagnosis. For intraperitoneal tumors, when a CT scan is not available, it is recommended that an MRI examination be performed to reach a definitive diagnosis. However, in the present case, the tumor was located behind the peritoneum, and was deeply seated and complex. In such cases, a preoperative MRI may not be able to make a definitive diagnosis due to the tumor being concealed by the stomach and other organs situated in front of it. Furthermore, an MRI scan is also associated with certain limitations: A relatively long examination time, a poor dynamic scanning effect of organ enhancement compared with CT, relatively high requirements regarding the patient's cooperation and the poor diagnosis of dehydrated organs and lesions; in addition, some patients, such as those with pacemakers, cannot be subjected to an MRI. Endoscopic ultrasound (EUS)-fine-needle aspiration (FNA) is a method of fine-needle puncture biopsy guided by endoscopic ultrasonography for the extraction of cells and tissue fragments for pathological examination, in order to help determine the nature of the lesion. Using this method, the pathological characteristics may be evaluated in detail, and pathological tissues may also be obtained for cytological and immunohistochemical examinations, in order to determine the nature, origin and classification of the lesion $(16,17)$. As a result, the initial treatment regimens often change. For medical units that have developed EUS-FNA technology, EUS-FNA can be improved according to the needs of the patients to further select specific treatment plans. Although EUS-FNA has a number of advantages, it may not be applied in all cases. In the present case, the tumor was close to the abdominal aorta, and it was deep-seated with limited operating field; furthermore, the risk of puncture associated with cystic lesions is higher compared with that for solid lesions. Therefore, in our patient, the preoperative incomplete MRI scan and EUS-FNA may have also contributed to the misdiagnosis.

Surgical resection remains the most effective treatment for retroperitoneal teratomas (18). Laparoscopic teratoma resection is safe and feasible due to its relatively advanced technology, minimal associated trauma, rapid patient recovery and preservation of capsule integrity of the mature cystic teratoma $(19,20)$. The complete resection of benign teratomas may prevent malignant transformation and recurrence (21). If the intraoperative fast frozen biopsy examination indicates malignancy, the surgical resection scope should be expanded. If the intraoperative tumor is found to invade the surrounding tissues or organs, multiple departments can cooperate to carry out multiple organ resection, supplemented by radiotherapy and chemotherapy to improve treatment efficacy $(22,23)$. In the present case, the combined findings of CT and other preoperative examinations could not rule out the possibility of an adrenal tumor, although GST was clinically suspected and despite the fact that there was no history of hypertension. Preoperative and intraoperative blood pressure monitoring revealed stable blood pressure, and an adrenal tumor was thus considered unlikely. However, for patients with irregular preoperative blood pressure, obvious intraoperative blood pressure changes and marked fluctuations, adrenal tumors should be considered in the differential diagnosis, and appropriate preoperative and intraoperative measures should be taken. 

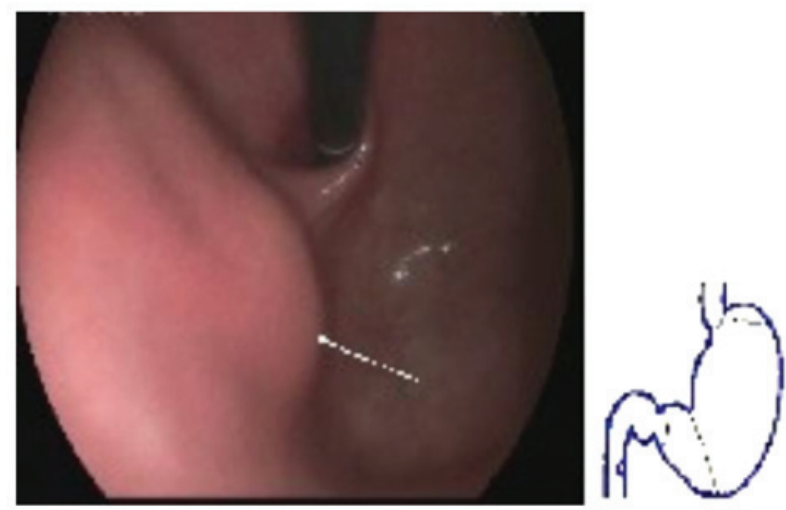

Figure 1. On electronic gastroscopy examination the gastric fundus mucosa was smooth, with a local bulge $\sim 2.5 \times 2.8 \mathrm{~cm}$ in size. Compared with upper gastroscopy, there was no major differences.

\section{A}

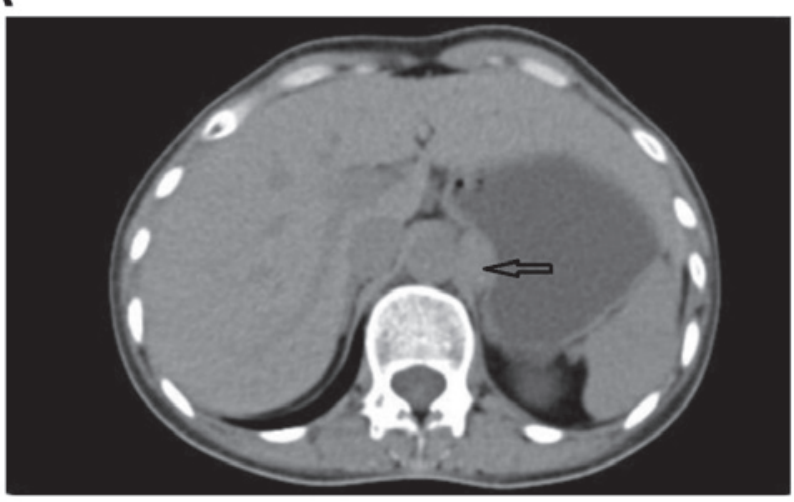

B

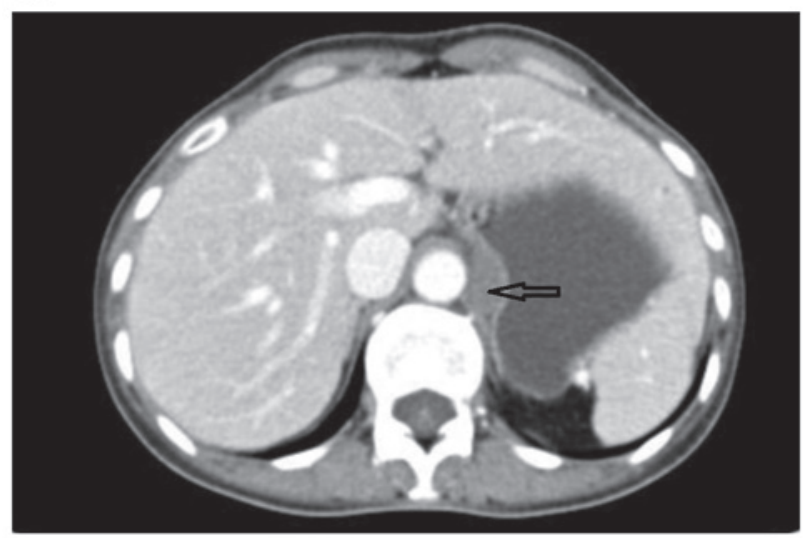

Figure 2. Dual-phase enhanced computed tomography of the small intestine. A mass (arrows) may be observed in the lesser curvature of the gastric fundus, with the lumen out of the lesion, with a clear margin, a size of $\sim 25 \times 18 \mathrm{~mm}$, surrounded by adipose tissue. Gastric stromal tumor was considered as a possible diagnosis. (A) CT plain scan; (B) CT contrast-enhanced.

A teratoma is a congenital neoplasm originating from germ cells. Therefore, for patients diagnosed with teratoma before or after surgery, an ultrasound examination of the reproductive system should be performed to avoid misdiagnosis. During follow-up in the present case, no reproductive teratoma was identified on gynecological ultrasound examination, although a regular follow-up was still recommended. Laboratory examinations of mature retroperitoneal teratomas are mostly

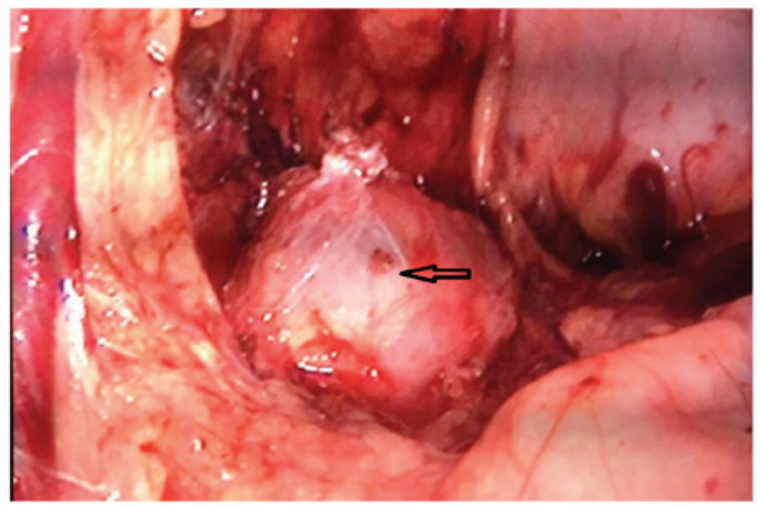

Figure 3. A 2x2-cm large cystic mass (arrow) was identified intraoperatively near the abdominal aorta and behind the peritoneum of the lesser curvature of the stomach.

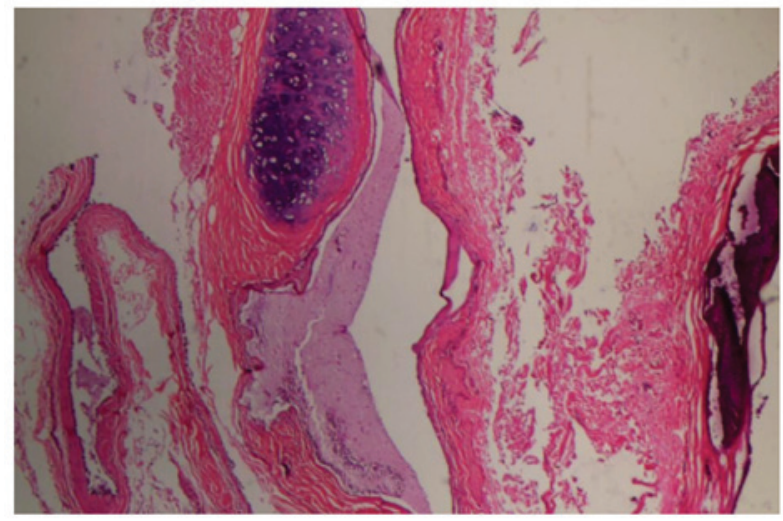

Figure 4. The results of the pathological examination confirmed the diagnosis of a retroperitoneal mature cystic teratoma.

negative. However, the increased levels of AFP, carcinoembryonic antigen (CEA) and human chorionic gonadotropin (HCG) may be of value for the diagnosis and prognosis of immature teratomas $(24,25)$. The AFP levels of this patient were mildly elevated, and the result of the pathological examination revealed a mature cystic teratoma. Although there were no obvious indications for radiotherapy and chemotherapy at the time, an abdominal CT examination, and measurement of AFP, CEA and hCG levels should be performed regularly after surgery to prevent tumor recurrence $(26,27)$.

In the present case, the tumors were similar to the gastric fundus of the lesser gastric curvature, with no obvious space between the tumor and the stomach as they are in close proximity, and it was difficult to detect on preoperative examination. Retroperitoneal tumors may be complex, and the tumor in the present case was deep-seated and fixed. The front surface of the tumor was also concealed by the gastric fundus, and there were no obvious changes observed with respiration and change in body position. Moreover, ultrasound gastroscopy, EUS-FNA, B-mode ultrasonography of the abdomen and MRI were not performed prior to surgery, and the histological type of the lesion could not be determined. In addition, this type of clinical case is rare, and the clinician's diagnostic experience was relatively insufficient, which is one of the main reasons for the misdiagnosis. 
In conclusion, retroperitoneal teratoma must be included in the differential diagnoses of GST, and may improve comprehensive learning. The aim of the present case report was to improve our current understanding of the clinical presentation of GSTs and retroperitoneal teratomas in order to reduce the likelihood of clinical misdiagnosis.

\section{Acknowledgements}

Not applicable.

\section{Funding}

The present study was supported by the key guiding project of Health and Health Committee of Hunan Province of China (grant no. 20201919 ).

\section{Availability of data and materials}

The datasets used and/or analyzed during the present study are available from the corresponding author on reasonable request.

\section{Authors' contributions}

LG was responsible for the conception, content, image collection and manuscript writing. QH was responsible for providing guidance with manuscript writing, revision and proofreading. Both authors contributed to the writing of the manuscript. Both authors have read and approved the final version of the manuscript.

\section{Ethics approval and consent to participate}

This case study was approved by the Ethics Committee of the First Affiliated Hospital, University of South China. The patient provided consent for inclusion in this study.

\section{Patient consent for publication}

Written informed consent was obtained from the patient for the publication the case details and any associated images.

\section{Competing interests}

The authors declare that they have no competing interests.

\section{References}

1. Pandit N, Awale L and Jaiswal LS: Giant calcified retroperitoneal teratoma. Indian J Surg Oncol 9: 436-437, 2018.

2. Huang X, Liu B and Xie L: Giant primary retroperitoneal teratoma in an adult female patient: A case report. Oncol Lett 6 : 460-462, 2013

3. Skinovsky J, Tsumanuma FK, Sigwalt MF, Panegalli-Filho F, Kawakubo AM, Rolim BG and Godoy LA: Thirty kilograms giant retroperitoneal teratoma: Case report. Arq Bras Cir Dig 29: 128-129, 2016 (In English).

4. Hul'ová S, Aziri R, Chovanec M, Mardiak J, Mego M and Pind'ák D: Clinical management and outcome in extreme retroperitoneal growing teratoma syndrome of testicular origin-clinical management and effect of the treatment. Klin Onkol 32: 129-132, 2019.

5. Richie JP: Re: Post-chemotherapy retroperitoneal teratoma in nonseminomatous germ cell tumors: Do predictive factors exist? Results from a national multicenter study. J Urol 199: 894, 2018.
6. Chalhoub K, Abou Zahr R, Mansour E, Aoun M and Jabbour M: Primary mature cystic teratoma compressing the prostate in a 28-year-old male: A case report and literature review. Case Rep Urol 2019: 8970172, 2019

7. Morotti A, Busso M, Consiglio Barozzino M, Cinardo P, Angelino V, Familiari U, Veltri A and Guerrasio A: Detection and management of retroperitoneal cystic lesions: A case report and review of the literature. Oncol Lett 14: 1602-1608, 2017.

8. Cvijanović R, Ivanov D and Zivojinov M: Thoracoabdominal teratoma-rare location. Med Pregl 64: 89-92, 2011 (In Serbian).

9. Li S, Liu Z, Dong C, Long F, Liu Q, Sun D, Gao Z and Wang L: Growing teratoma syndrome secondary to ovarian giant immature teratoma in an adolescent girl: A case report and literature review. Medicine (Baltimore) 95: e2647, 2016.

10. Maenosono R, Saito K, Ibuki N, Takahara K, Inamoto T, Nomi H and Azuma H: A case of retroperitoneal teratoma difficult to distinguish from adrenal tumor. Hinyokika Kiyo 63: 525-528, 2017 (In Japanese).

11. Tong HX, Liu WS, Jiang Y, Liu JU, Zhou JJ, Zhang Y and $\mathrm{Lu}$ WQ: Giant retroperitoneal bronchogenic cyst mimicking a cystic teratoma: A case report. Oncol Lett 9: 2701-2705, 2015.

12. Ma Y, Zheng J, Feng J, Zhu H, Xiao X and Chen L: Ectopic nephrogenic rests in children: A series of 13 cases in a single institution. Pediatr Blood Cancer 65: e26985, 2018.

13. Lee KH, Song MJ, Jung IC, Lee YS and Park EK: Autoamputation of an ovarian mature cystic teratoma: A case report and a review of the literature. World J Surg Oncol 14: 217, 2016.

14. Goel S, Aeron R, Goel A and Singhai A: Retroperitoneal teratoma simulating giant adrenal myelolipoma: A diagnostic puzzle. BMJ Case Rep 2017: pii: bcr-2017-221762, 2017.

15. Cheng W, Qi Y, Wang B, Tian L, Huang W and Chen Y: Characteristics and computed tomography evaluation of primary retroperitoneal tumours: Report of 113 cases. Ann R Coll Surg Engl 99: 55-59, 2017.

16. Val-Bernal JF, Martino M, Terán A, Yllera E and Castro-Senosiain B: Endoscopic ultrasound-guided fine-needle aspiration cytology in the diagnosis of leiomyomas of the gastrointestinal tract. Rev Esp Patol 52: 154-162, 2019.

17. Matsumoto K, Takeda Y, Onoyama T, Kawata S, Kurumi H, Koda $\mathrm{H}$, Yamashita $\mathrm{T}$ and Isomoto $\mathrm{H}$ : Endoscopic ultrasound-guided fine-needle aspiration biopsy-Recent topics and technical tips. World J Clin Cases 7: 1775-1783, 2019.

18. Kriger AG, Berelavichus SV, Son AI, Gorin DS, Ikramov RZ and Kalinin DV: Robot-assisted operations for non-organ retroperitoneal tumors. Khirurgiia (Mosk): 24-28, 2015 (In Russian).

19. Lv Z, Bai X, Sheng Q, Liu J and Wu Y: A case report of a giant mature teratoma of the thyroid gland in a young girl. Medicine (Baltimore) 98: e14703, 2019.

20. Li H, Zhao T, Wei Q, Yuan H, Cao D, Shen P, Liu L, Zeng H and Chen N: Laparoscopic resection of a huge mature cystic teratoma of the right adrenal gland through retroperitoneal approach: A case report and literature review. World J Surg Oncol 13: 318, 2015.

21. Jones VS and Burns CR: Operative considerations in pediatric retroperitoneal teratomas-a review. Eur J Pediatr Surg 23: 265-269, 2013.

22. Narasimhan V, Smith M, Claydon M, Wale R and Warrier S: Incidental large retroperitoneal teratoma in a patient with colorectal carcinoma. ANZ J Surg 88: 1350-1352, 2018.

23. Kim JH, Lee TS, Oh HK and Choi YS: A case of mucinous adenocarcinoma arising from retroperitoneal teratoma treated with chemoradiation. J Gynecol Oncol 20: 126-128, 2009.

24. Seike T, Kagaya T, Komura T, Nakai R, Shimizu Y, Omura H, Ohta H, Ohyama S, Kawashima A and Unoura M: A rapidly growing, large, mature retroperitoneal teratoma in an adult male patient. Nihon Shokakibyo Gakkai Zasshi 114: 1008-1014, 2017 (In Japanese).

25. Sasi W, Ricchetti GA, Parvanta L and Carpenter R: Giant mature primary retroperitoneal teratoma in a young adult: Report of a rare case and literature review. Case Rep Surg 2014: 930538, 2014.

26. Hoang VT, Trinh CT, Le TB and Le TK: Recurrence of retroperitoneal mature cystic teratoma in an adult: A case report. Radiol Case Rep 14: 692-696, 2019.

27. Li J, Gong P, Liu F, Sun P and Wu C: Retroperitoneal cystic immature teratoma: A case report. Oncol Lett 10: 1023-1025, 2015. 